This document is published in:

Robotics and Computer-Integrated Manufacturing (2010), 26(2), 119-129.

DOI: $10.1016 /$ j.rcim.2009.08.001

(C)2009 Elsevier Ltd.

This work has been supported by the CAM Project S2009/DPI-1559/ROBOCITY2030 II, developed by the research team RoboticsLab at the University Carlos III of Madrid. 


\title{
Design issue of a new iCub head sub-system
}

\author{
Nestor Eduardo Nava Rodríguez \\ Robotics Lab, Carlos III University, Calle Universidad, 30, 28911 Leganes (Madrid), Spain \\ E-mail address: nnava@ing.uc3m.es
}

\begin{abstract}
I am presenting a new design for the head sub-system of the humanoid robot iCub. ICub is a humanoid robot platform that has been conceived as a result of the development of the European Project RobotCub. Mechanical problems of the current head sub-system of iCub robot have been studied in order to figure out improvements for a new design. The feasibility of the new design has been checked through dynamic simulations of the head operations. The successful results have shown plots with curve evolutions of smooth shapes and suitable values of important kinematic and dynamic parameters of the head structure.
\end{abstract}

Keywords: Robotics, Humanoid robot, ICub, Head sub-system, Design

\section{Introduction}

In humanoid robot projects, the head sub-system represents an important element since it provides the robot-environment interaction through the gesture and vision systems. Several robot head projects have been developed in research centres and universities around the world. One example is the CeDAR system [1] developed at Australian National University. CeDAR (Cable Drive Active Vision Robot) is a fully cable-driven system for active vision application. CeDAR incorporates a common tilt axis and two independent pan axes separated by a baseline. The system achieves the human vision system, although it carries two fully motorized zoom digital cameras weighing $350 \mathrm{~g}$ each. It is lightweight and incurs minimal deflection under high acceleration for added precision.

ASP (Agile Stereo Pair) [2] is another example of a successful vision system. It has been developed by the Computer Vision and Systems Laboratory (CVSL) with the collaboration of the Robotics Laboratory of the Mechanical Engineering Department of Laval University. This architecture involves two rapid, precise and independent cameras that enable 3D vision. A 2-degree of freedoms (DOF) parallel manipulator performs the orientation of each camera. This mechanical architecture leads to high velocities and accelerations with low cost and compact design.

CASIMIRO [3] is a device of a perceptual user interface, developed at University of Las Palmas de Gran Canarias, which makes the interaction between people and computers easier. This architecture is based on the scheme of semantic fusion. To achieve this goal, the interface has human-like behaviors that are based on a humanoid head with facial movements. The perceptual side of the interface makes use of an active vision approach.
The M4 project-Macaco [4] consists of the development of a biologically inspired monkey. This four-legged biologically inspired robot will have some degree of intelligence, using an active vision head to perceive the environment. The Leg Laboratory at the Artificial Intelligence Lab of MIT has developed the legs and the Humanoid Robotics Group has been developing an active vision head. The head design currently includes a neck and two eyes. The neck has three revolute actuating joints with three DC motors. The active vision head is composed of four video cameras actuated by motors at each joint. The eyes have a common actuated tilt joint and independent pan actuated joints. Each eye has two cameras to simulate human vision. The top cameras are $\mathrm{B} / \mathrm{W}$ high-resolution cameras that acquire images within a small field of view. The two lower cameras are color low-resolution cameras that acquire images of a large field. Other significant projects of robotic head are reported in [5], such as Maveric head from USC; Cramiun from Waseda University; and Lulabot from JPL, as well as the head of the ARMAR humanoid robot from the University of Karlsruhe [6].

Paper I will show a design process of a new head for the humanoid Robot iCub [7]. ICub is a humanoid robot that has been created as an open platform freely available to research in embodied cognition. Several design problems have appeared during the prototype operation of the current head. Solutions to those problems have been proposed for the design of the new head. Suitable results of dynamic simulations validate the feasibility of the new design. 


\section{Design problems of the current iCub head sub-system}

The initial specifications for the iCub head sub-system demand the anatomic characteristics of a three-and-a-half year-old child in terms of dimensions and weight. Fig. 1(a) shows an overview of the whole current structure of the iCub prototype. Fig. 1(b) shows in detail a built iCub head with the current design. Fig. 1(b) shows a 3D-CAD model developed in PTC Pro/Engineer Wildfire 2 environment [8]. In order to achieve a good replication of the human movements, six degrees of freedoms (DOF) can be recognized in this sub-system with the rages of movements as follows:
The current mechanical design of the iCub head is divided in three main parts: neck, eye and cover [9]. The neck sub-system is a 3-DOF serial manipulator, placed in a configuration that properly simulates the human neck movement. Fig. 2(a) shows in detail a neck joint of the current iCub head formed by a Faulhaver DC motor [10] with planetary gearbox.

Table 1 contains a list of the specifications of the actuation for the three roll, swing and pan DOF of iCub head. Note that the structures of all iCub neck joints are quite similar and only differ in the motor specifications. Although the current neck design is

- $1 \mathrm{DOF}$ for roll of neck, moving from $-50^{\circ}$ to $40^{\circ}$;

- $1 \mathrm{DOF}$ for swing of neck, moving from -40 to $40^{\circ}$;

- 1 DOF for each pan of eye; moving from $-45^{\circ}$ to $45^{\circ}$;

- 1 common DOF for tilt of eye, moving from $-40^{\circ}$ to $40^{\circ}$.

a

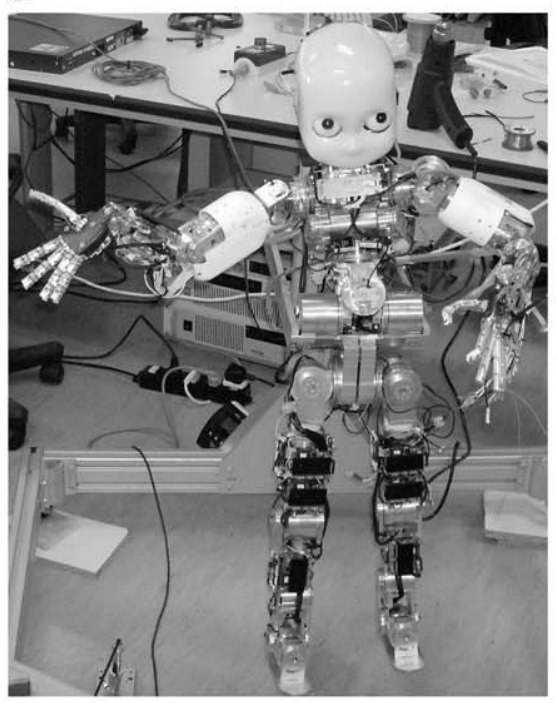

b

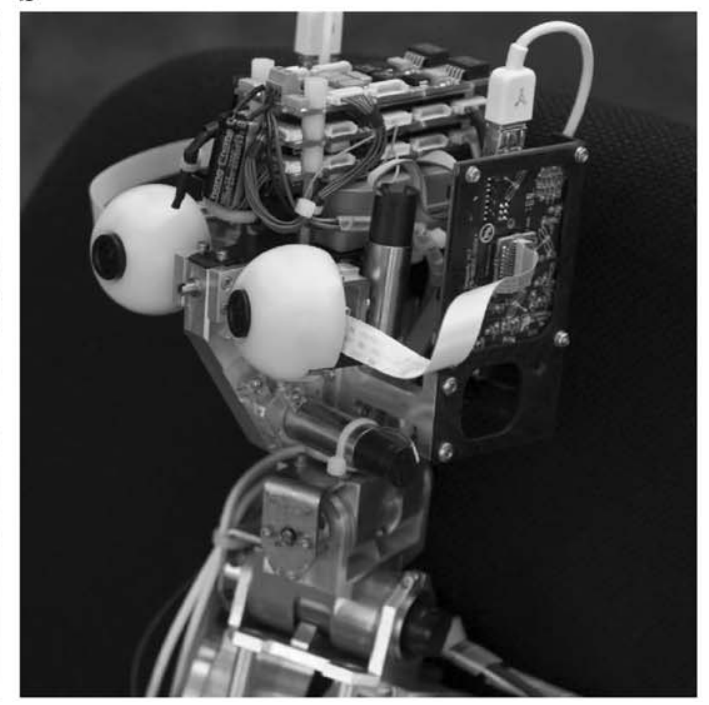

C

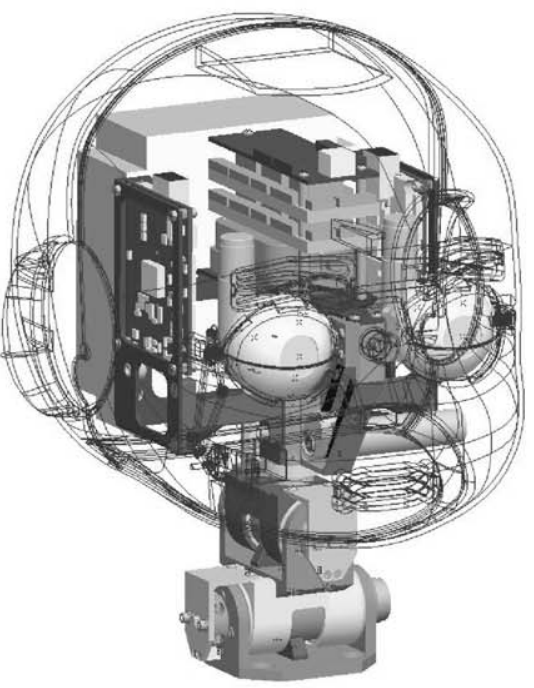

Fig. 1. The current design of iCub head sub-system: (a) a built prototype of the whole structure, (b) a detail of the head sub-system and (c) a 3D-CAD model.

a

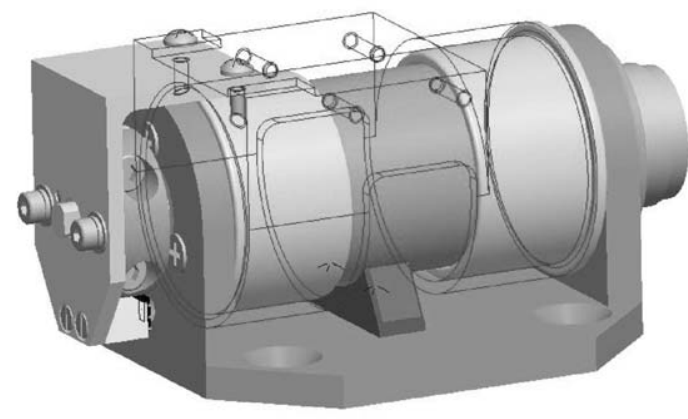

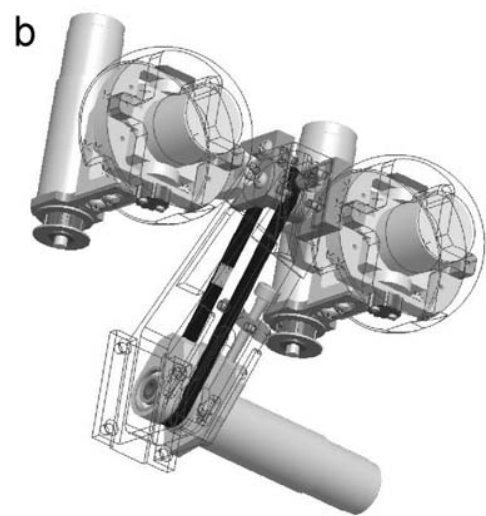

Fig. 2. Details of the components of current iCub head of Fig. 1: (a) neck joints (bushes, motor cover, joint base. Joint connection is translucent) and (b) eye sub-system (common tilt motor, pan motors, belt-actuating mechanism, video cameras. Nose and eye balls are translucent). 
robust, easy to operate and highly performing, it presents the following problems:

- The motors barely fit the torque requirements for the head movement operation. A torque quite bigger than that just required for a free robot movement is necessary in some control applications.

- Neck joints contain bushes, as shown in Fig. 2(a), that generate high friction, leading to loss of mechanical energy.

- The high reduction ratios of the current planetary gearboxes result in low velocities, as shown in Table 1, that do not fulfill the robot application requirements.

- The current planetary gearboxes present some backlash that penalizes the robot accuracy.

The eye mechanism has a serial configuration with 3 DOF. Both eyes can pan independently by using 1 DOF for each one and they can tilt simultaneously using a common DOF. Fig. 2(b) shows in detail the eye mechanism of the current iCub head. Each DOF of the eye is actuated remotely by a Faulhaber DC motor with a planetary gearbox, as shown in Table 1, through a belt mechanism. The pan movement is driven by a belt mechanism with the motor located at the eye ball. The tilt is actuated by a belt mechanism located between the eyes. Each belt has a tension adjustment mechanism. For the necessary acceleration and speed, the DC motors equipped with optical encoders have comfortably larger specifications than required. Nevertheless, the current iCub eye sub-system presents the following design problems:

- The belt system presents slippage and backlash since the force is transmitted by the contact between belt teeth.

- In highly dynamic applications, the belt system can generate some vibrations, unsuitable for the vision system, which require frequent adjustment of tension.

- The fact that the tilt motor has to carry the pan motors slows the system down.

- A small amount of backlash is always necessary to reduce excessive wear, heat and noise created by the current gearboxes.

In humanoid robots, some of the main design issues are features meant to facilitate communication with humans. The current iCub

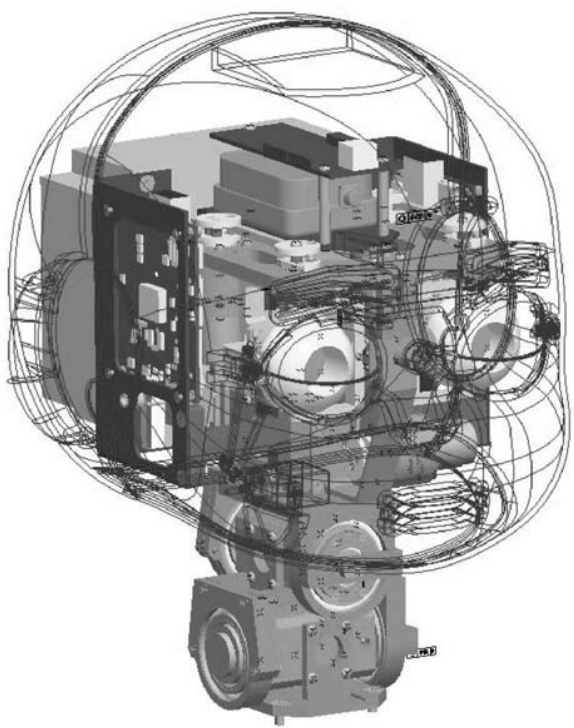

Fig. 3. A 3D-CAD model of the new iCub head design.

head wears an external cover, as shown in Fig. 1(b), which contributes to human-computer interaction. The external cover also ensures the protection of the head mechanism, absorbing external hits that the robot may suffer during operation. In the first prototype of iCub head, toy-like features have been selected for the design.

\section{Solutions for a new design of icub head sub-system}

Taking into account the above-mentioned problems of the current iCub head, a new sub-system has been designed in order to improve the robot mechanical structure and performance. Fig. 3 shows a 3D-CAD model of the new iCub head design, still divided into three parts: neck, eye and cover.

In the new design, the cover has the same toy-like structure of the current head design. The neck sub-system is still configured in a serial 3-DOF manipulator with the joints located in the same form as the current design. In the new design, the bushes have

Table 1

Actuation data for the iCub head sub-system.

\begin{tabular}{|c|c|c|c|c|c|c|}
\hline \multirow{2}{*}{$\frac{\text { Description }}{\text { Motor }}$} & \multicolumn{2}{|l|}{ Roll and swing } & \multicolumn{2}{|l|}{ Pan } & \multicolumn{2}{|l|}{ Eye } \\
\hline & Faulhaber (current) & Faulhaber & Faulhaber (current) & Faulhaber & Faulhaber (current) & Faulhaber \\
\hline Code & 1724 & 2342 & 1717 & 2224 & 1319 & 1717 \\
\hline Shall torque (Nm) & 0.013 & 0.092 & 0.0054 & 0.022 & 0.0029 & 0.0054 \\
\hline Velocity (rpm) & 8200 & 8100 & 14000 & 8100 & 13100 & 14000 \\
\hline$D_{\max }(\mathrm{mm})$ & 17 & 23 & 17 & 22 & 13 & 17 \\
\hline Length (mm) & 24 & 42 & 17 & 24.2 & 32.3 & 17 \\
\hline Weight $(\mathrm{g})$ & 27 & 88 & 18 & 46 & 12 & 18 \\
\hline Gear Head & GYSIN & Harmonic Drive & Faulhaber & Harmonic drive & Faulhaber & Harmonic drive \\
\hline Code & GPL 22R 3-stage & HFUC-5-100-2A-R-SP & 16/7-246:1 & HFUC-5-100-2A-R-SP & 14/1-246:1 & HFUC-5-50-2A-R-SP \\
\hline Ratio & $343: 1$ & $100: 1$ & $246: 1$ & $100: 1$ & $246: 1$ & $50: 1$ \\
\hline Weight (g) & 100 & 11.4 & 33 & 11.4 & 20 & 11.4 \\
\hline $\mathrm{D} \max (\mathrm{mm})$ & 22 & 20 & 17 & 20 & 14 & 20 \\
\hline Length max (mm) & 52 & 11.4 & 29.4 & 11.4 & 48.2 & 11.4 \\
\hline Rated torque (Nm) & 3 & 0.11 & 0.45 & 0.11 & 0.45 & 0.11 \\
\hline Max Speed (rpm) & 6000 & 10000 & 5000 & 1000 & 5000 & 10000 \\
\hline Efficiency (\%) & 85 & 59.4 & 60 & 59.4 & 80 & 59.4 \\
\hline Output torque (Nm) & 3.79 & 5.43 & 0.80 & 1.31 & 0.57 & 0.16 \\
\hline Output Speed (rpm) & 20.32 & 48.11 & 34.15 & 48.11 & 42.60 & 166.32 \\
\hline
\end{tabular}



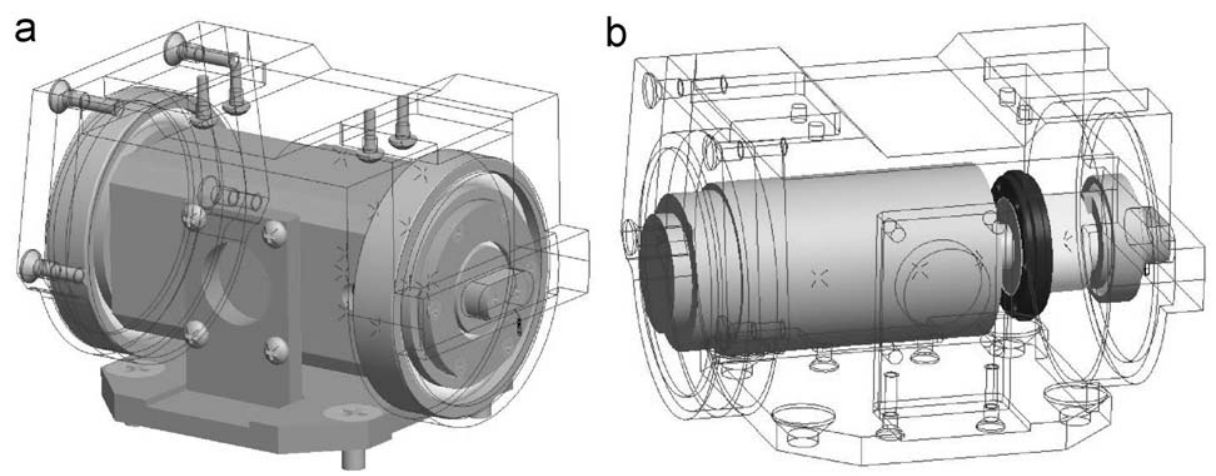

Fig. 4. Neck joint details of the new iCub head design: (a) joint structure (bearings and motor cover. The joint connection is translucent) and (b) motor+Harmonic Drive assembly (the whole joint structure is translucent).

been replaced by bearings in order to reduce the friction, as shown in Fig. 4(a), which details the neck joint structure. The neck joint actuations are composed of Faulhaber DC motor with Harmonic Drive [11], as shown in Fig. 4(b). Table 1 contains a list of the specifications of the new joint motors and Harmonic Drives. As can be seen in Table 1, the joint velocities and torques have been increased as a result of the new actuation assembly. Moreover, Harmonic Drives show low backlash, which provides accuracy of the system. The new neck design is robust, accurate and easy to operate and also can perform human-like movements for the iCub head operation.

Fig. 5 shows the new design of the iCub eye sub-system. The new eye is a parallel manipulator of 2 DOF in which the tilt is still a common DOF and the pan is an independent DOF for each eye.

The serial configuration of the current eye design has been changed to a parallel configuration since the parallel manipulator has high speed and is robust and also provides proper human-like eye movement. The eye DOF are still actuated remotely by Faulhaber DC motors with Harmonic Drives in a similar configuration as the neck joint actuation. Moreover, the Harmonic Drives provide low backlash and reduce the weight of the system. Table 1 contains the details of the new eye actuation. Four-bar linkage mechanisms transmit the motion from the motors to the eye moving platform, as shown in Fig. 6. Fig. 6(a) shows the articulated mechanism that drives the movement for the common eye tilt. As in the current head design, this mechanism is still between the eyes. Fig. 6(b) shows the articulated mechanism that drives the movement for the eye pan. Each eye has its own driving articulated mechanism since individual pan actuation is still used for the new eye design. The articulated mechanisms are low backlash, rigid and robust. Those characteristics are suitable for a vision system.

\section{Dynamic simulations of the new iCub head design}

By using the 3D-CAD model of Fig. 3, simulations of the new iCub head have been examined in Pro/Engineer environment in order to validate the feasibility of the assembly and check the operation under dynamic conditions. Mechanism Toolbox of Pro/Engineer [8] has been used for dynamic simulations due to the convenient features for simulating the operation of multibody systems that have been modelled in Pro/Engineer. The integrator is an algorithm that solves the differential equations of a dynamic problem over an internal of time during a simulation. The Mechanism Toolbox of Pro/Engineer used computes backward differentially the algebraic equations of the dynamic model.

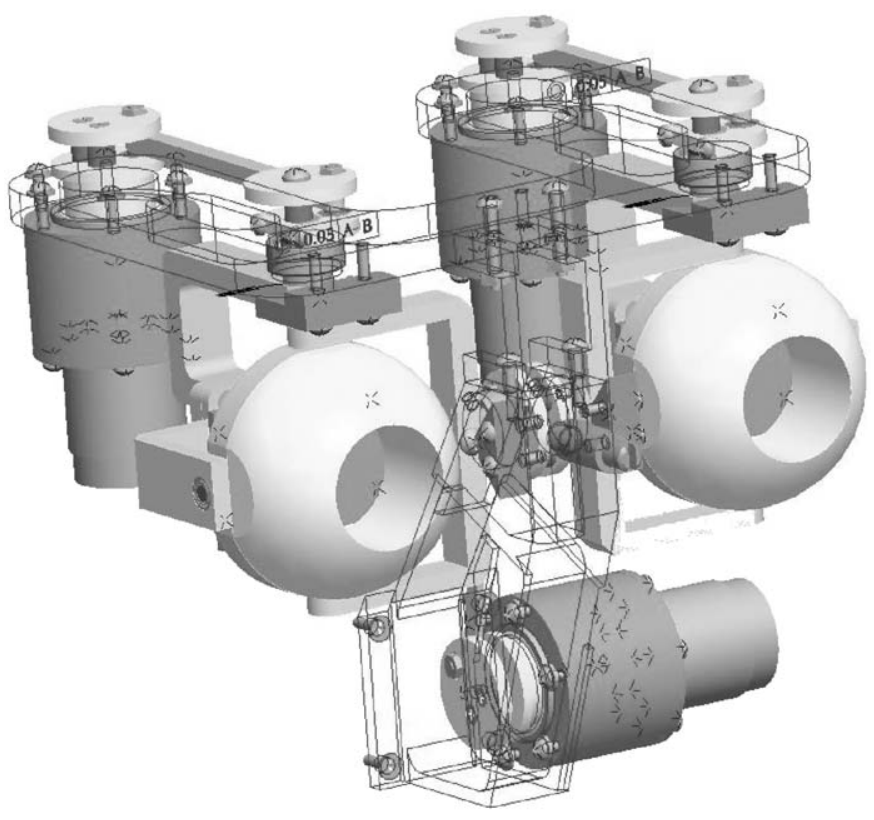

Fig. 5. Eye sub-system of the new iCub head design (tilt mechanism and pan mechanism. The sub-system supports are translucent.)

The iCub head model in Pro-Engineer environment takes into account several aspects such as external forces, gravity, contact constraints, friction and inertia properties. Friction effects are assumed negligible for the iCub joints in the simulation since lowfriction bearings [12] have been used in the robot design.

The model has been elaborated by introducing each component with its specific characteristics in terms of material, mass, density, shape and mechanical design. Almost all iCub head components have been modelled as made of aluminium alloy. Components, such as the cover and electronic boards, have been modelled by representing their material properties as accurately as possible. The dynamic simulations involve the simulated iCub in two modes. The neck and eye operations have been simulated separately since, as mentioned before, the model considered has been divided into neck, eye and cover. A first mode of dynamic simulation is related to a neck operation in which all 3-DOF are actuated. Fig. 7 shows position, velocity and acceleration plots that have been set up as neck motor inputs during the simulations. Note that in Fig. 7 solid lines represent the roll joint inputs, dotted lines represent the swing joint inputs and dashed lines represent the pan joint inputs. The second mode of 


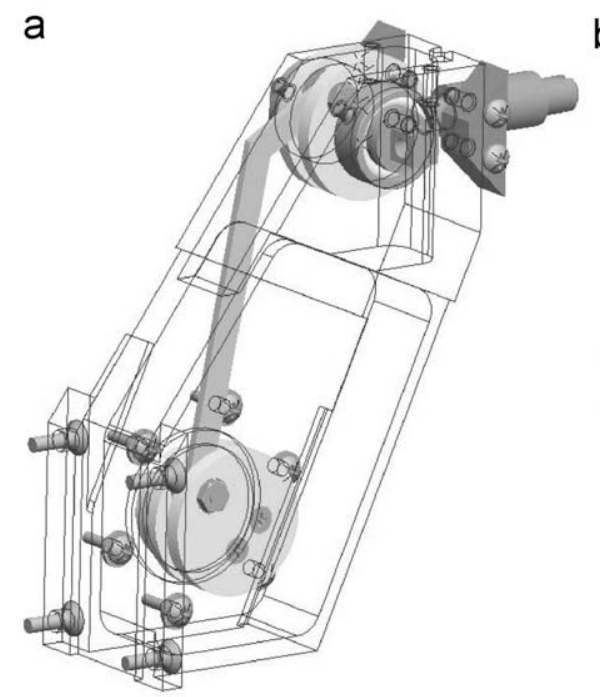

b

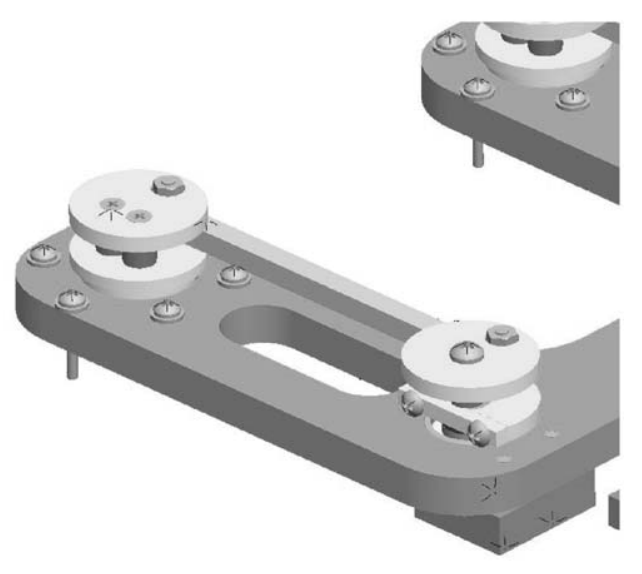

Fig. 6. Detail of the articulated mechanisms that drive the eye sub-system: (a) tilt driving mechanism (crank, follower and output link. The mechanism support is translucent.) and (b) pan driving mechanism (support, crank, follower and output link).

a

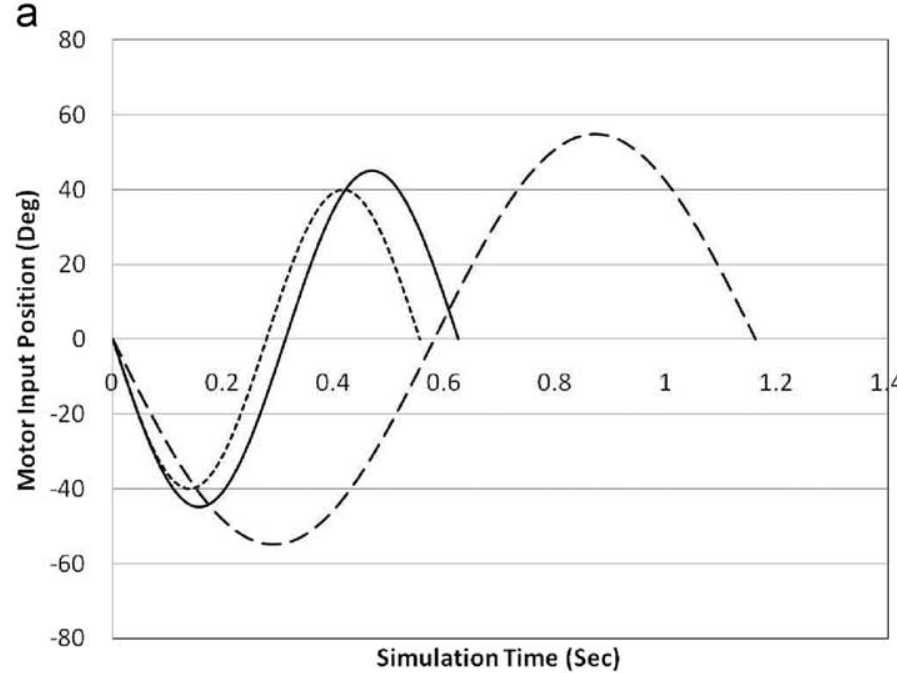

b

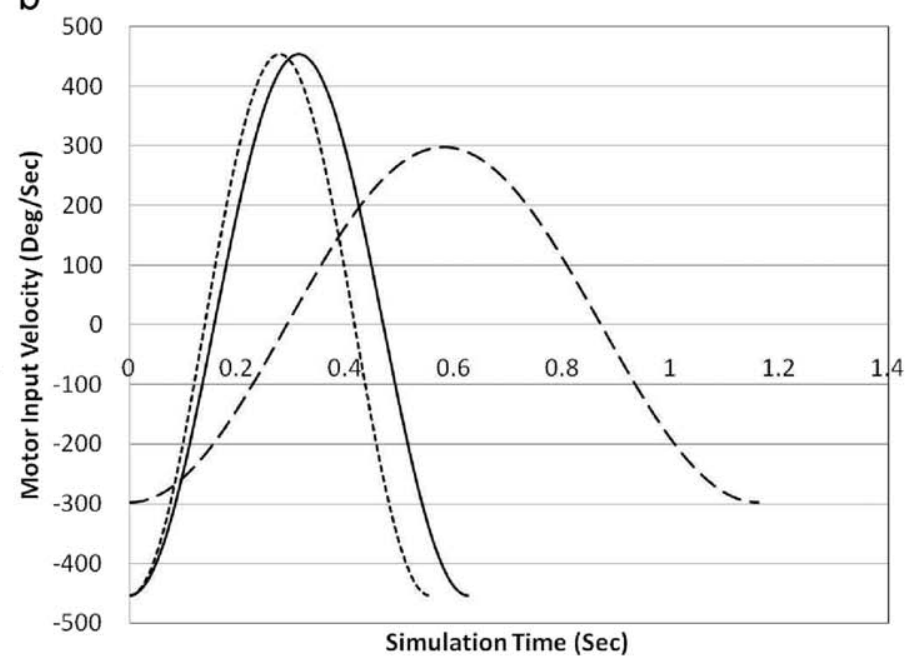

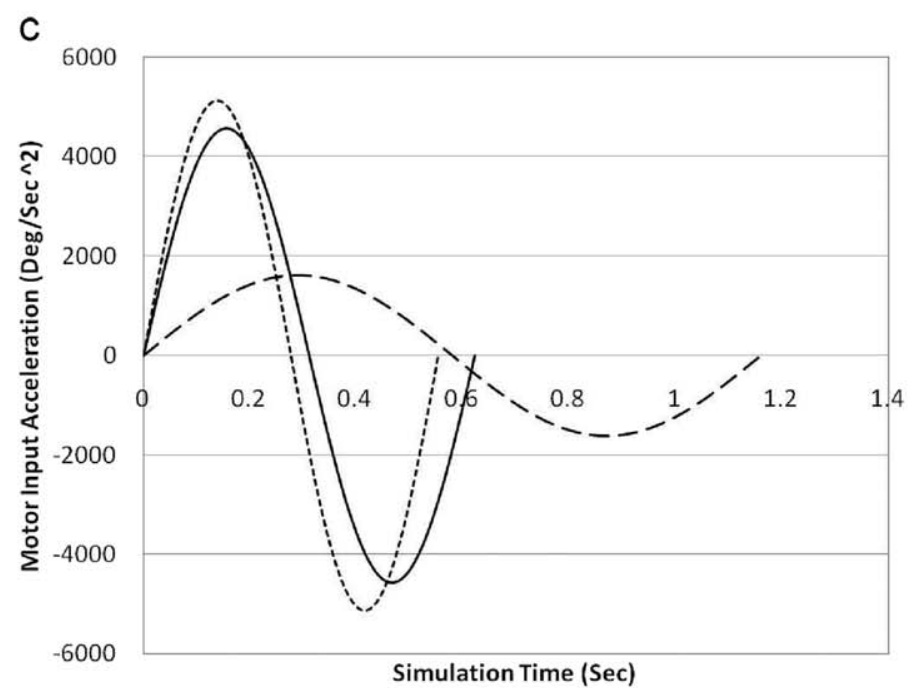

Fig. 7. Motor input for the dynamic simulation of the neck sub-system operation: (a) position, (b) velocity and (c) acceleration (roll in continues line; swing in dotted line; pan in dashed line). 
dynamic simulation is related to eye operation. In this case, the tilt joint is actuated from $-45^{\circ}$ to $45^{\circ}$, the left pan joint is actuated from $-45^{\circ}$ to $45^{\circ}$ and the right pan joint is actuated from $45^{\circ}$ to $-45^{\circ}$. Fig. 8 shows the input plots of position, velocity and acceleration of eye motors during the dynamic simulation. Note that the inputs are the same for all three eye actuators. Nevertheless, the input time history of right pan joint is offset $180^{\circ}$ in relation to the others. The time interval of $0.01 \mathrm{~s}$ (100 intervals in $1 \mathrm{~s}$ ) has been selected for the dynamic simulations in order to obtain suitable results within reasonable computational periods. The total simulation time was $2.00 \mathrm{~s}$.

\section{Numerical results of the dynamic simulations}

Fig. 9 shows a sequence of the first simulated mode during the iCub neck operation. Fig. 10 shows the plots of position, velocity and acceleration of the neck joints obtained during the simulation. Note that in Fig. 10 the roll curves are displayed as solid lines, the swing curves are displayed as dotted lines and the pan curves are displayed as dashed lines. These output plots show curves with a harmonic evolution that match the input plots of

a

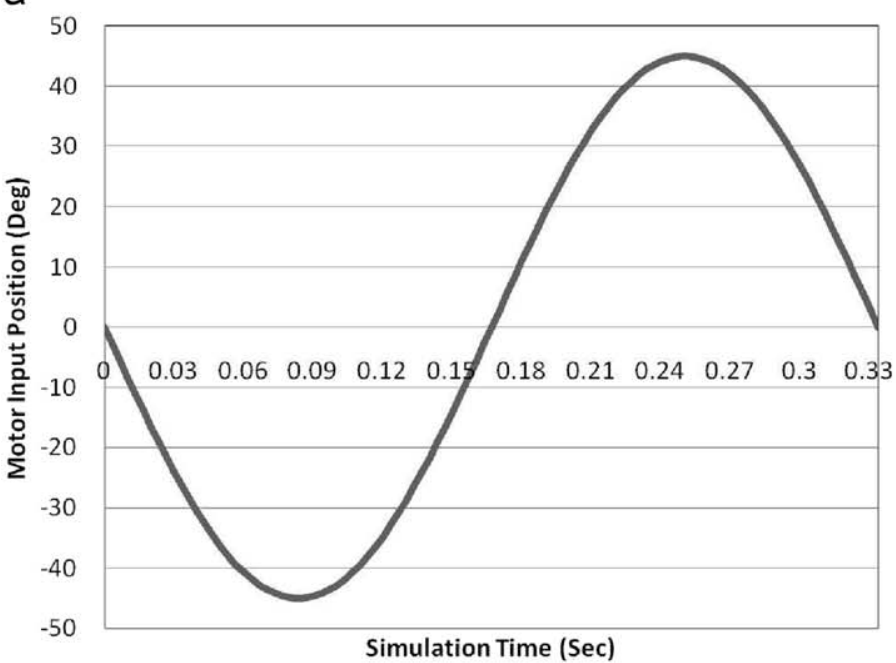

Fig. 7. The position plot of Fig. 10(a) shows harmonic curves with movement ranging from $-45^{\circ}$ to $45^{\circ}$ for the roll joint, $-40^{\circ}$ to 40 for swing joint, and $-55^{\circ}$ to $55^{\circ}$ for pan joint of neck. The velocity plot of Fig. 10(b) displays harmonic curves with moment ranging from $-450^{\circ}$ to $450^{\circ} \mathrm{s}$ for roll and swing joints and $-300^{\circ}$ to $300 \% \mathrm{~s}$ for pan joint of neck. The acceleration plot of Fig. 10(c) contains harmonic curves with movement ranging from $4500^{\circ}$ to $-4500^{\circ} / \mathrm{s}^{2}$ for roll joint, $5000^{\circ}$ to $-5000^{\circ} / \mathrm{s}^{2}$ for swing joint, and $2000^{\circ}$ to $-2000^{\circ} / \mathrm{s}^{2}$ for pan joint of neck. For the new iCub head sub-system, I expect that a built prototype will present the same accurate and no-backlash behavior of the simulation. Fig. 11 shows the torque plots for the 3-DOF of neck, which have been obtained during the dynamic simulation of Fig. 9. Fig. 11(a) shows the time history of the actuating torque of neck roll joint. This curve presents a harmonic evolution with a range from -2.5 to $2.5 \mathrm{~N} \mathrm{~m}$. Similarly, the torque curve of swing joint of Fig. 11(b) has a harmonic evolution with a range from about -1.5 to about $1.5 \mathrm{Nm}$.

Fig. 11(c) shows the harmonic evolution of the torque curve of pan neck joint that is contained in a range of about -0.2 to about $0.2 \mathrm{~N} \mathrm{~m}$. Note that the motors selected for the neck, listed in Table 1 , can fulfill the torque requirements of Fig. 11 obtained

b

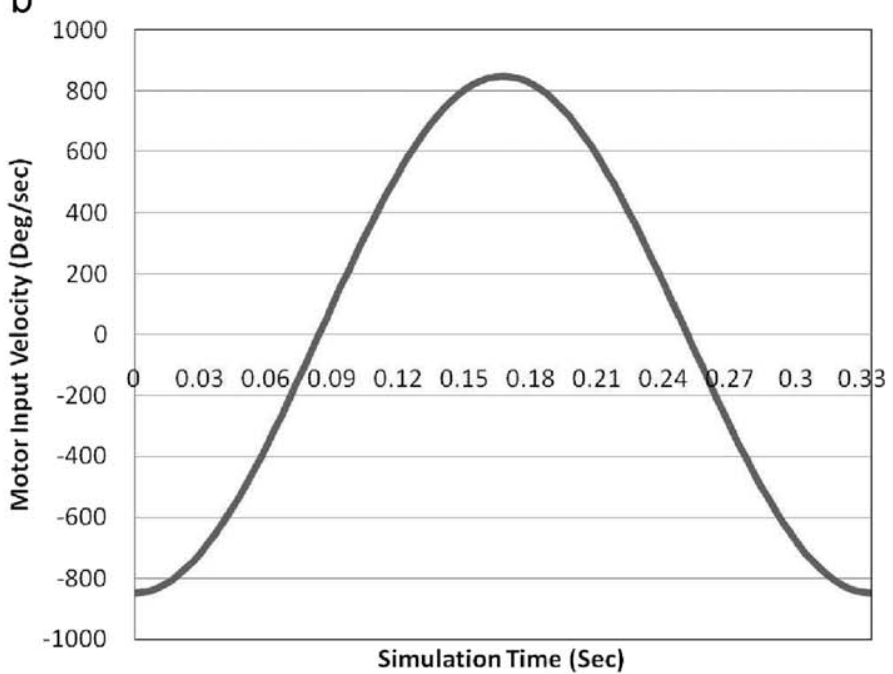

C

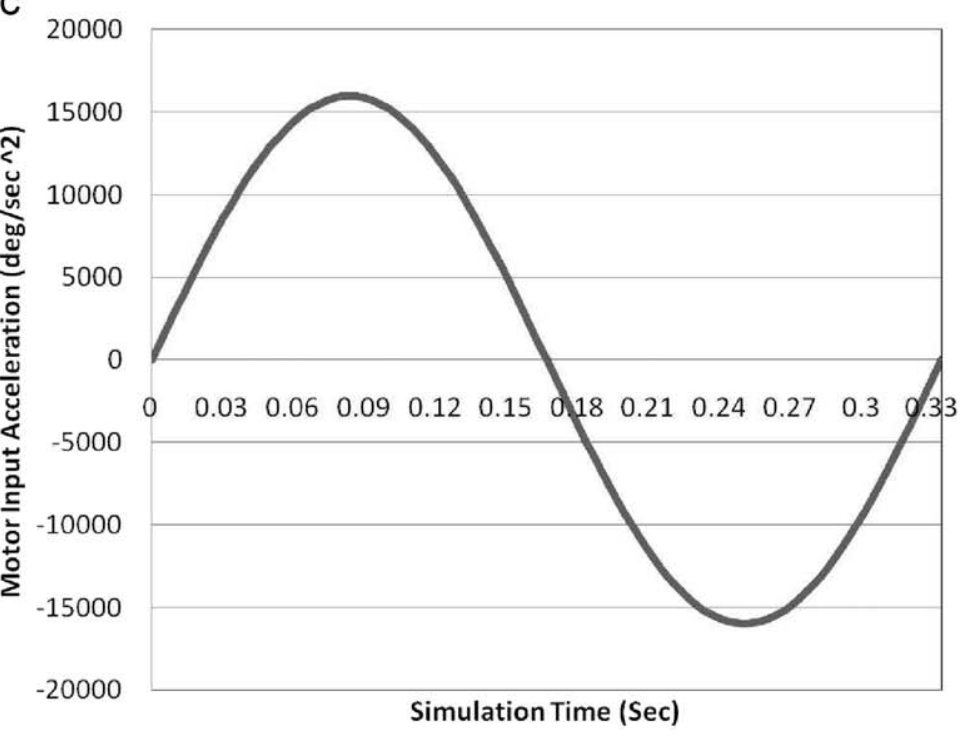

Fig. 8. Motor input for the dynamic simulation of the eye sub-system operation: (a) position, (b) velocity and (c) acceleration. 

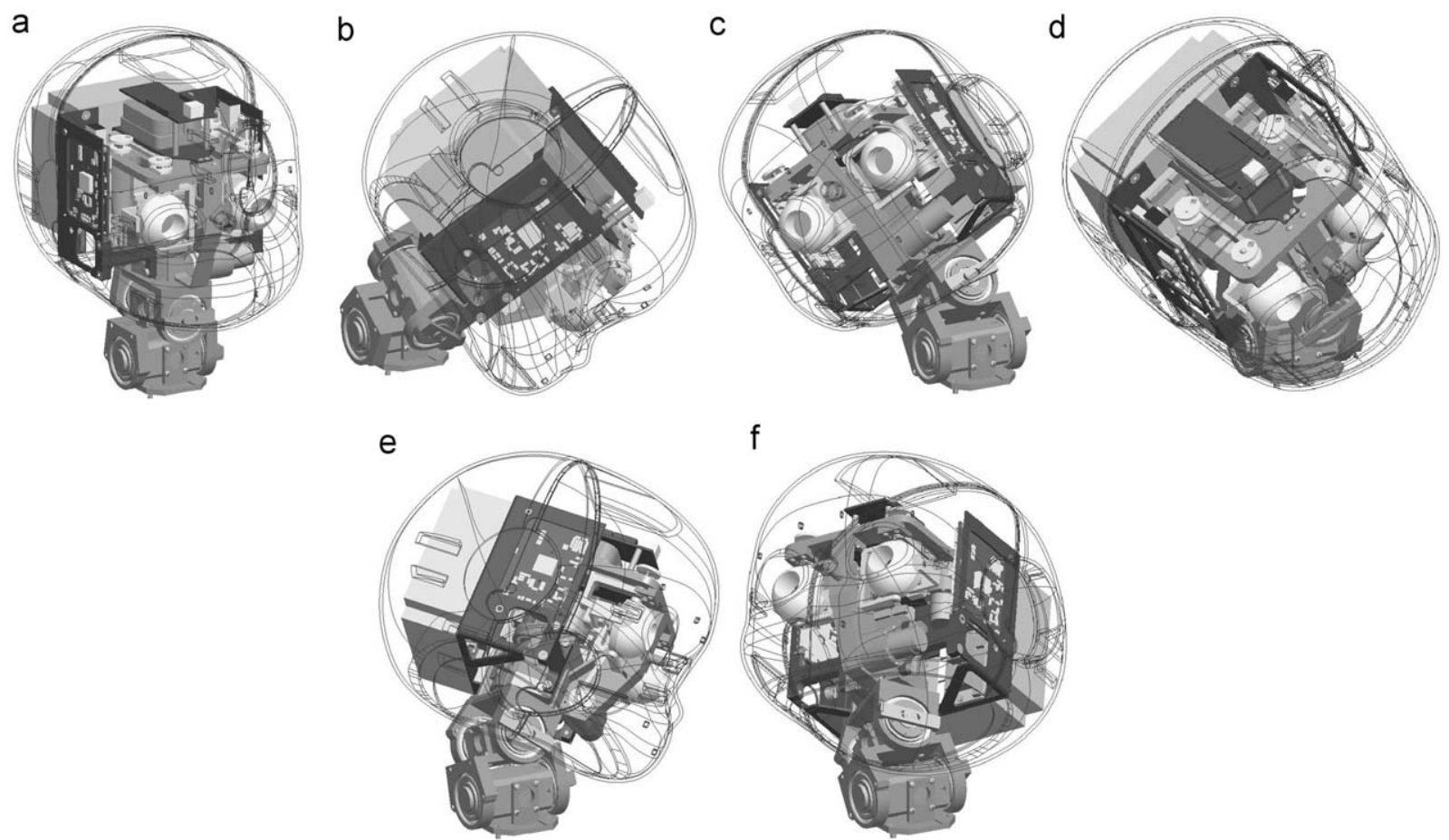

Fig. 9. A sequence of the dynamic simulation for the neck sub-system operation: (a) at $0.00 \mathrm{~s}$ of simulation time, (b) after $0.40 \mathrm{~s}$ of simulation time, (c) after $0.80 \mathrm{~s}$ of simulation time, (d) after $1.20 \mathrm{~s}$ of simulation time, (e) after $1.60 \mathrm{~s}$ of simulation time and (f) after $2.00 \mathrm{~s}$ of simulation time.
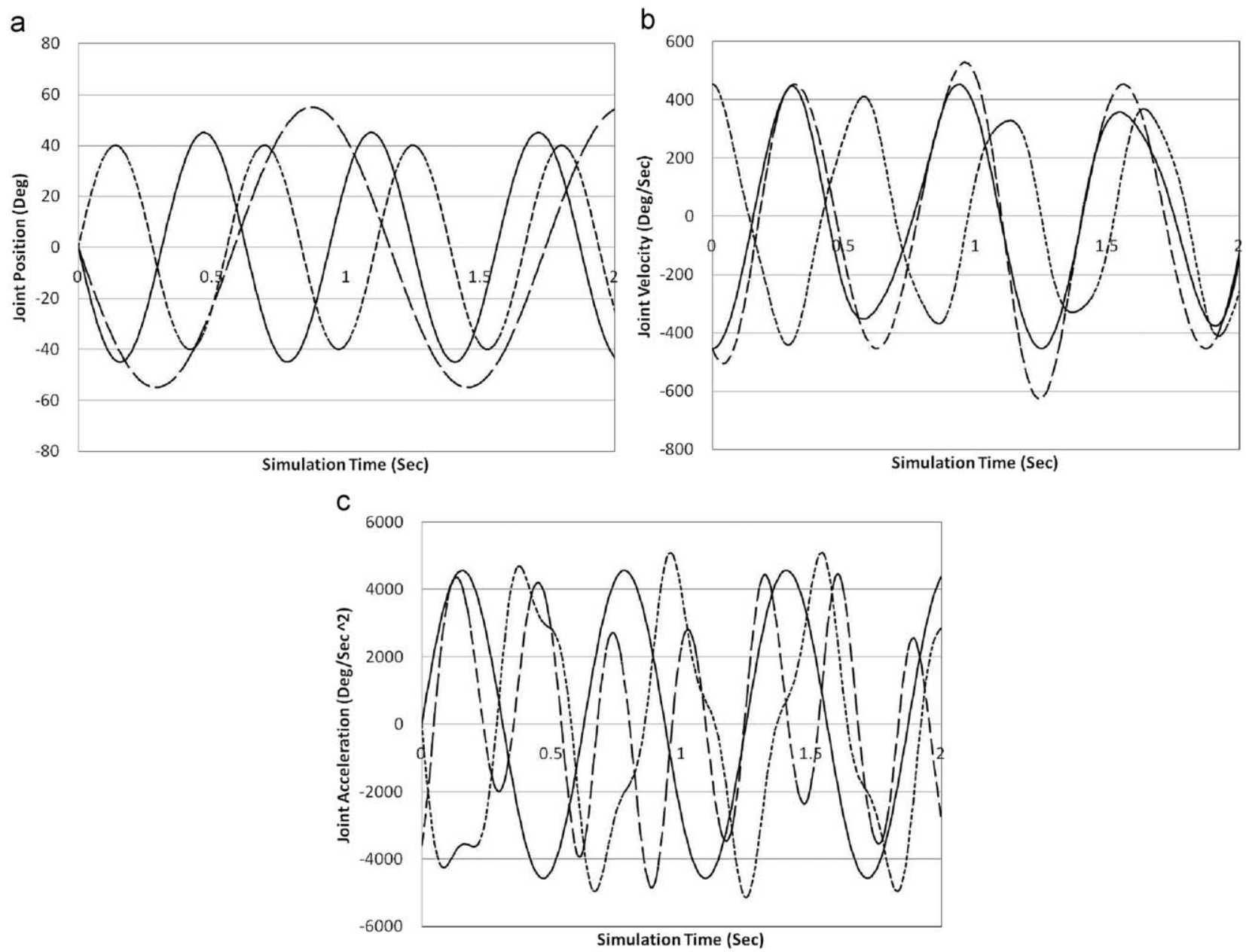

Fig. 10. Time history of joint motion of the neck sub-system: (a) position, (b) velocity and (c) acceleration (roll in solid line; swing in dotted line; pan in dashed line). 

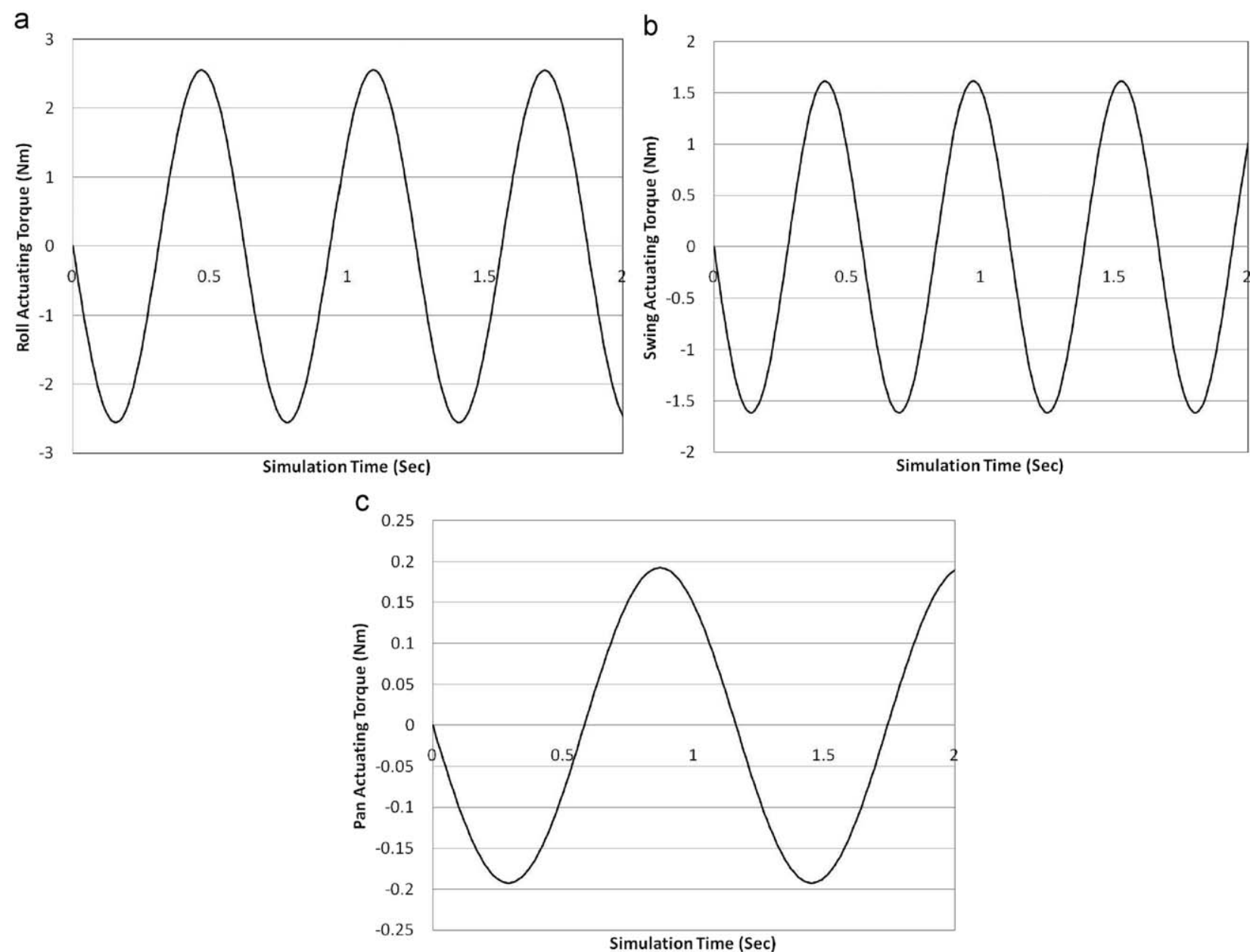

Fig. 11. Time history of actuating torque of the neck sub-system: (a) roll actuation, (b) swing actuation and (c) pan actuation.

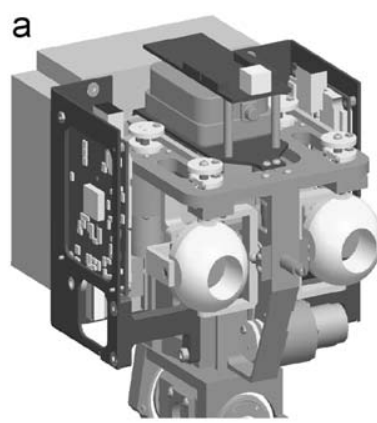

b

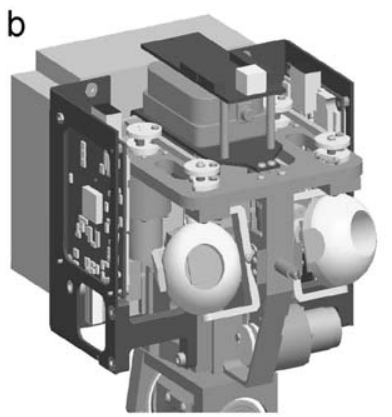

e

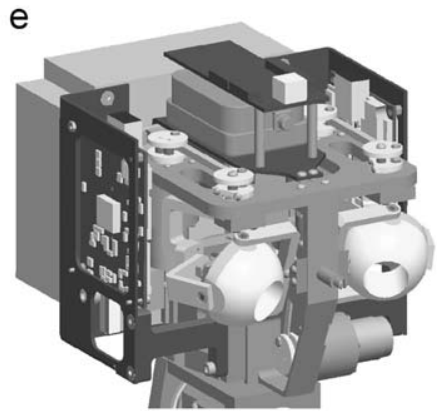

C

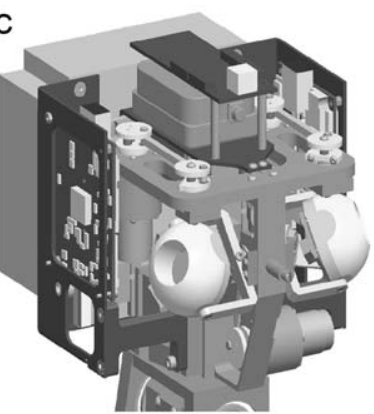

d

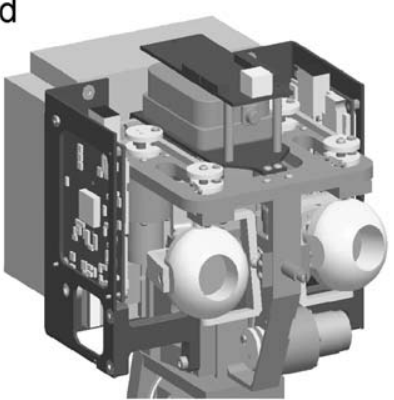

f

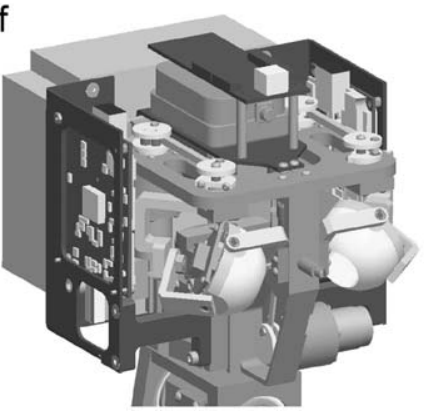

Fig. 12. A one-cycle sequence of the dynamic simulation for the eye sub-system operation: (a) at $0.00 \mathrm{~s}$ of simulation time, (b) after $0.04 \mathrm{~s}$ of simulation time, (c) after $0.08 \mathrm{~s}$ of simulation time, (d) after $0.15 \mathrm{~s}$ of simulation time, (e) after $0.21 \mathrm{~s}$ of simulation time and (f) after $0.25 \mathrm{~s}$ of simulation time. 
during the simulation. For example, the output torque of the selected actuation for swing neck joint that has been listed on the third column of Table 1 is $5.43 \mathrm{Nm}$.

Fig. 12 shows a sequence obtained during the dynamic simulation of the second mode of the iCub eye operation. Figs. 13-15 show plots of some significant results that have been obtained during this dynamic simulation. Fig. 13 shows the plots for position, velocity and acceleration of eye pan movement. The solid lines represent the motion curves of output shaft of pan joint and the dotted lines represent the curves of pan motion of eye platform. Similar to the neck simulation results, the motion curves of Fig. 13 match the eye actuation inputs of Fig. 8. In fact, the position values of the output shaft of the pan eye joint are in a range from $-45^{\circ}$ to $45^{\circ}$, velocity values are in a range from about $-800 \%$ s to about $800 \%$ s, and acceleration values are in a range from about $-15,000^{\circ} / \mathrm{s}^{2}$ to about $-15,000^{\circ} / \mathrm{s}^{2}$. Nevertheless, the parallel manipulator of iCub eye sub-system performs an offset in the pan movement of the moving platform with respect to the input shaft, as shown by the curves of Fig. 13. The tilt movement induces small displacements in the pan direction during simultaneous tilt-pan operations that generate the abovementioned pan offset. Indeed, when the eye performs only pan movement the plots for position, velocity and acceleration are the same as the output shaft of pan.

During a functioning of the new iCub head prototype, the encoder controller of the pan motor will compensate this pan offset for an accurate operation of the robot. This is possible since the curve evolutions of Fig. 13 show harmonic and repetitive shapes that permit a not-so-complex programming of the controller. In the case of the eye tilt, the position, velocity and acceleration of the mechanism output shaft and the eye moving platform have the same curve evolution and values, as shown in Fig. 14.

Fig. 14 shows the time history of position, velocity and acceleration of the eye tilt. Fig. 14(a) shows the position plot that goes from $-45^{\circ}$ to $45^{\circ}$, Fig. 14(b) shows the velocity plot that goes from $-800^{\circ}$ to $800^{\circ} / \mathrm{s}$, and Fig. 14(c) shows the acceleration plot that goes from $-15,000^{\circ}$ to $15,000^{\circ} / \mathrm{s}^{2}$. Similar to the other cases, the tilt motion plots of Fig. 14 correspond to the tilt input of Fig. 8. High velocities and accelerations are suitable characteristics for the eye motion since they provide high-speed performance for the moving platform.

Fig. 15 shows the plots of actuating torques of the eye DOF. Fig. 15(a) shows the actuating torque of tilt and Fig. 15(b) shows a

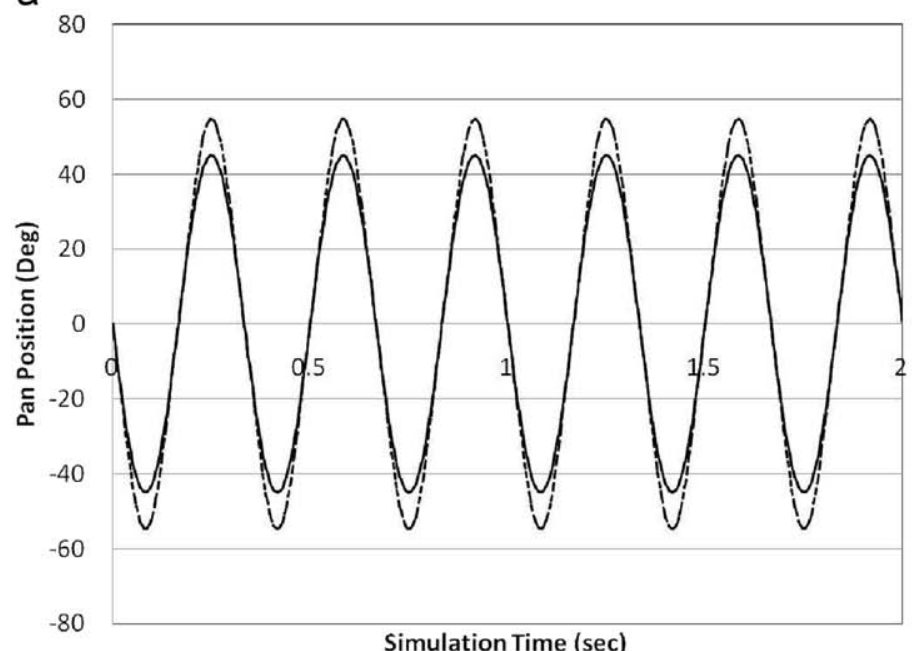

b

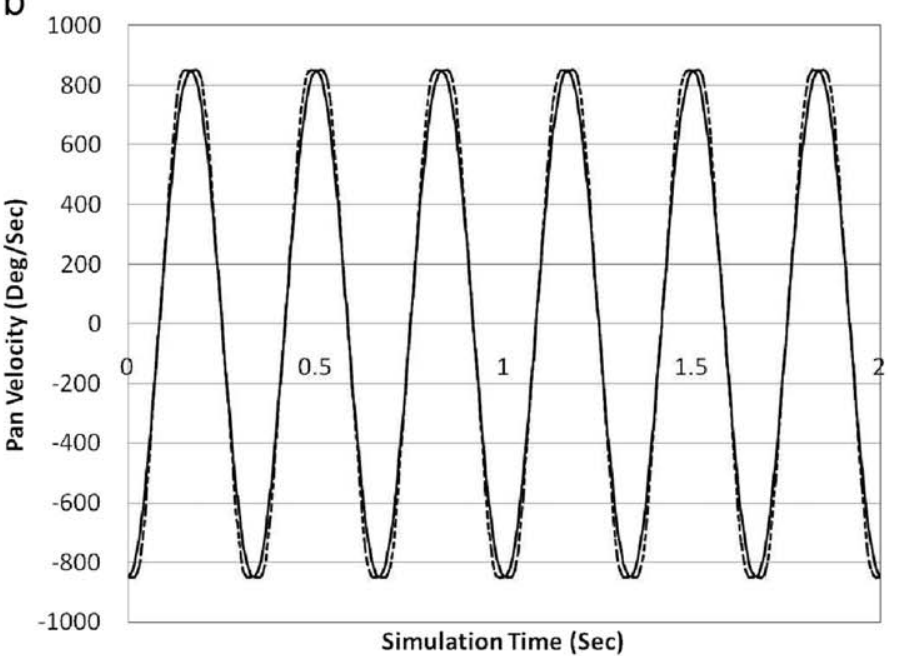

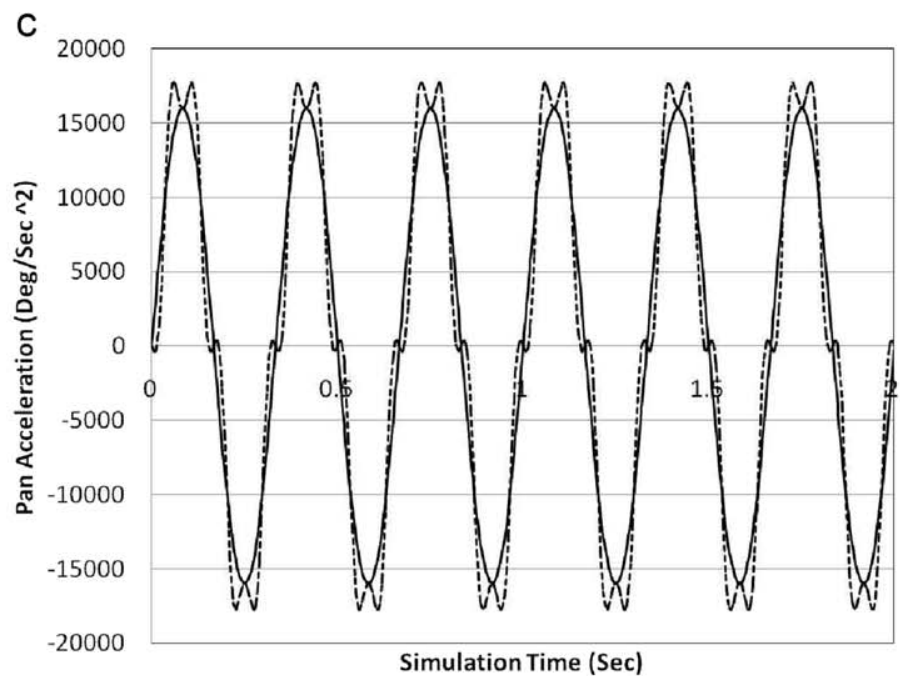

Fig. 13. Time history of pan motion of the eye sub-system: (a) position, (b) velocity and (c) acceleration (motor output shaft in solid line; eye moving platform in dotted line). 
a

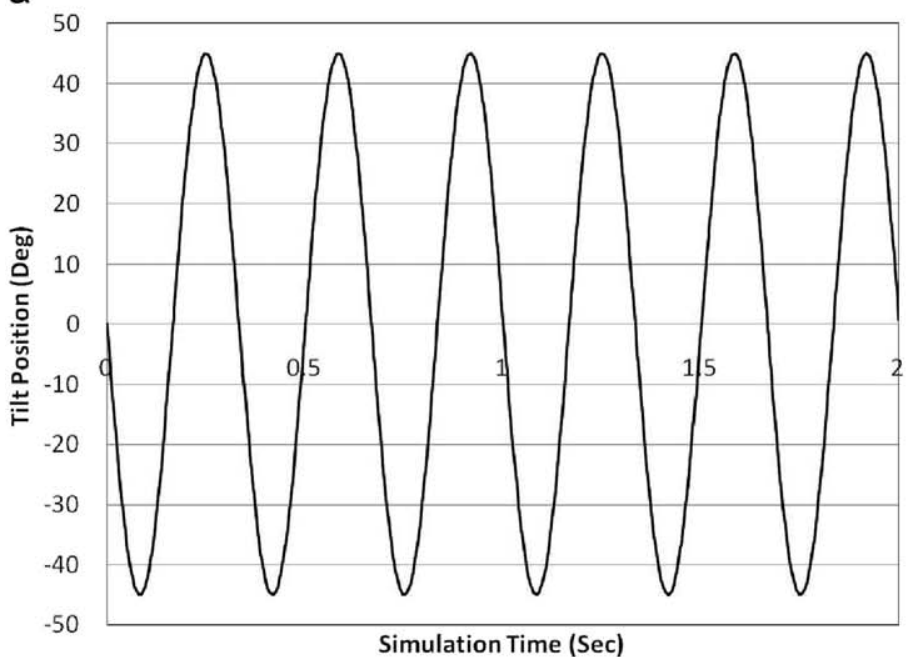

Simulation Time (Sec) b

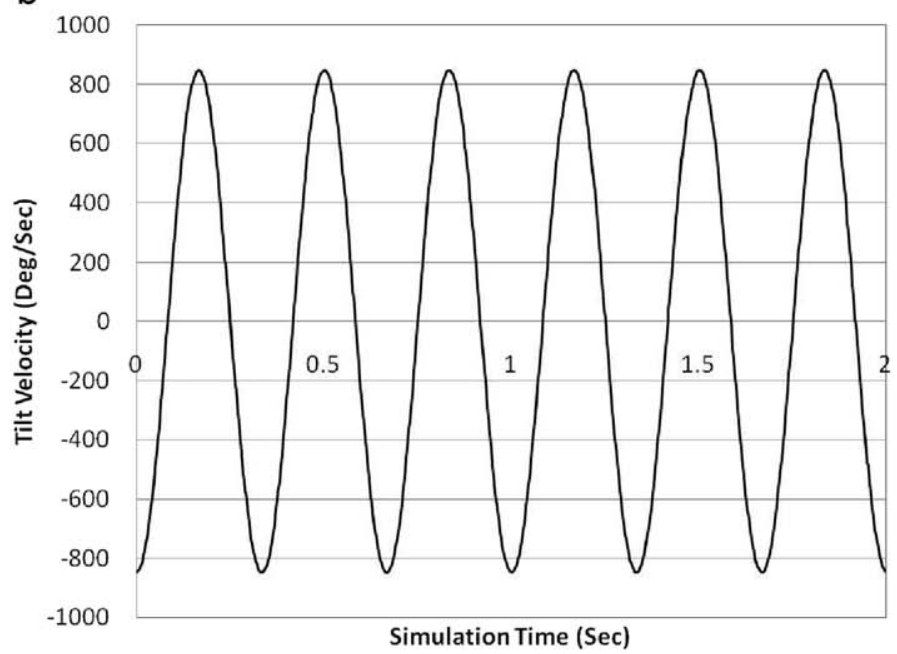

C

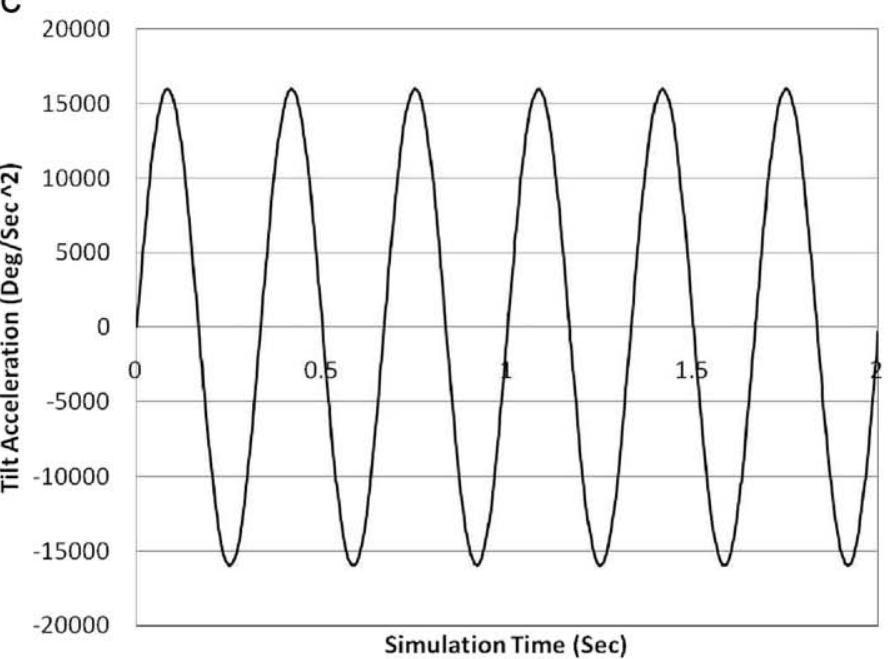

Fig. 14. Time history of tilt motion of the eye sub-system: (a) position, (b) velocity and (c) acceleration.

a

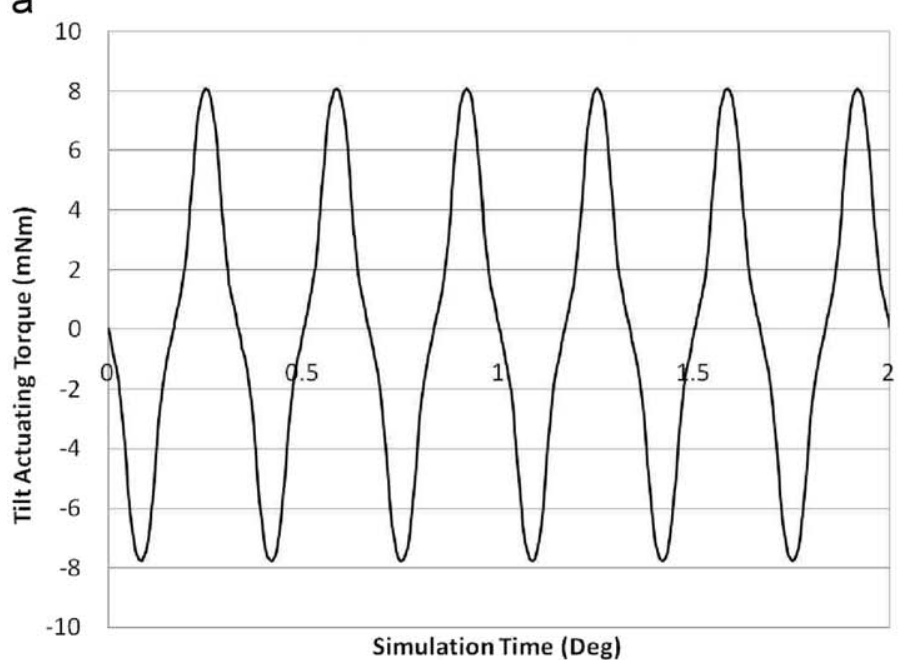

b

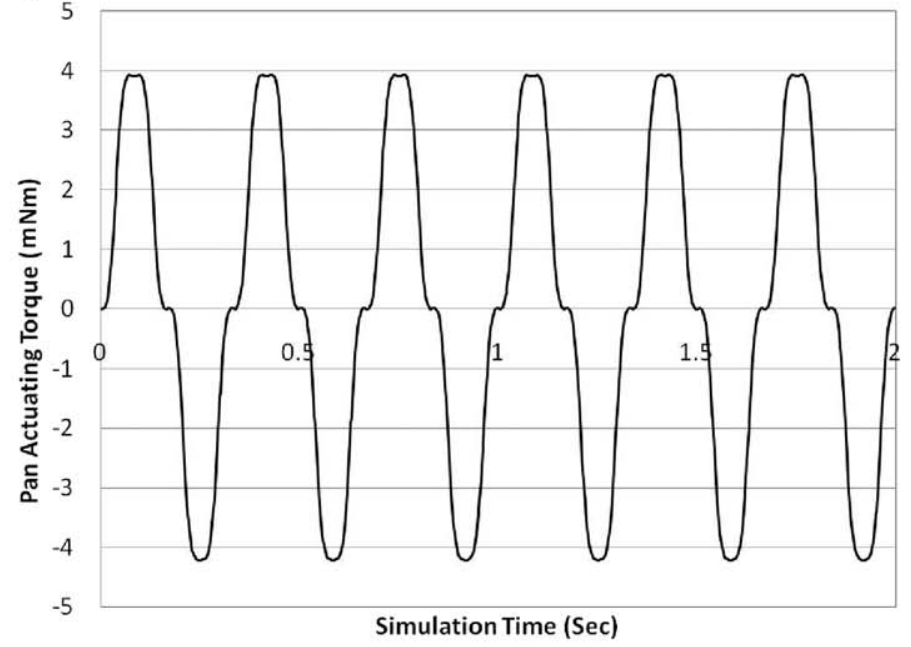

Fig. 15. Time history of actuating torque of the eye sub-system: (a) tilt actuation and (b) pan actuation.

the actuating torque of pan. Note that the curve evolutions of both actuating torques of pan have the same shape, but with a $180^{\circ}$ offset, as in the case of the motor input motion. The maximum actuating torque obtained during the dynamic simulation has been about $8 \mathrm{mN} \mathrm{m}$ for the tilt and $4 \mathrm{mN} \mathrm{m}$ for the pan of the eye. Similar to the neck case, these torque requirements for eye 
actuation can be fulfilled comfortably by the selected actuation for the new eye that is listed on the last column of Table 1 .

\section{Conclusions}

A new design has been illustrated for the head sub-system of iCub robot. Structural improvements have been proposed since the current design has shown problems during operation. The current problems of head design can be summarized mainly as low torque and velocity, backlash and excessive friction. New actuations, in term of motors and gearboxes, have been proposed for the actuating joints in order to increase torque and velocity and decrease backlash. Bearings have been used in the head joints in order to reduce friction. The new proposed structure provides performance enhancements for the iCub head sub-system. Dynamic simulations have been done in order to check the operation of the new head design. To validate operation, significant simulation results have been computed and analysed. Curve evolutions and values have been considered in the study of the simulation results. Smooth curves, cyclic evolutions and suitable values have been common characteristics of the simulation results. The proper results of the dynamic simulations have validated the new head design.

\section{References}

11] Truong $\mathrm{H}$, Abdallah $\mathrm{S}$, Rougeaux $\mathrm{S}$, Zelinsky A A novel mechanism for stereo active vision. In: Proceeding of Australian conference on robotics automation ACRA. Melbourne: ARAA; 2000.

[2] Samson E, Laurendeau D, Parizeau M, Comtois S, Allan J-F, Gosselin C. The agile stereo pair for active vision. Machine Vision and Applications Journal 2006;17(1):32-50.

[3] Deniz O, Castrillon M, Lorenzo J, Guerra C, Hernandez D, Hernandez M. CASIMIRO: a robot head for human-computer interaction. In: Proceeding of international conference on robot and human interactive communication. Berlin, 2002. p. 319-24.

[4] Arsenio A. M4 Project: Adding an Active Vision Had to the M4 Robot. Report to DARPA; March 2000.

[5] Android Head Robot Homepage. 〈http://www.androidworld.com/prod04. htm $>; 2008$.

[6] Asfour T, Welke K, Azad P, Ude A, Dillmann R. The karlsruhe humanoid head. Daejeon: Humanoids; 2008 p. 447-53.

[7] Sandini G, Metta G, Verson D, Caldwell D, Tsagarakis N, Beira R, et al. The robotcub project an open framework for research in embodied cognition. Tsukuba: Humanoids; 2005.

[8] Smith SG. Pro/ENGINEER Wildfire 3.0 update manual for wildfire 2.0 users, 2008.

[9] Beira R, Lopes M, Praca M, Santos-Victor J, Bernardino A, Metta G, et al. Design of the robot-Cub (iCub) head. In: Proceeding of international conference of robotics and automation ICRA. Orlando, 2006. p. 94-100.

[10] Faulhaber Homepage. DC-Motors. 〈www.faulhaber-group.com〉, 2008.

[11] HD homepage. 〈www.harmonicdrive.com〉, 2008.

[12] SKF homepage. 〈www.skf.com〉, 2008. 